\title{
Diffuson-mediated thermal and ionic transport in superionic
}

\section{conductors}

Tim Bernges ${ }^{[a]}$, Riley Hanus ${ }^{[b]}$, Bjoern Wankmiller ${ }^{[a, c, d]}$, Kazuki Imasato ${ }^{[\mathrm{e}]}$, Siqi Lin $^{[\mathrm{f}]}$,

Michael Ghidiu $^{[\mathrm{a}]}$, Marius Gerlitz ${ }^{[\mathrm{g}]}$, Martin Peterlechner ${ }^{[\mathrm{g}]}$, Samuel Graham ${ }^{[\mathrm{b}]}$, Geoffroy

Hautier $^{[\mathrm{h}, \mathrm{i}]}$, Yanzhong Pei ${ }^{[\mathrm{f}]}$, Michael Ryan Hansen ${ }^{[\mathrm{c}]}$, Gerhard Wilde ${ }^{[\mathrm{g}]}$, G. Jeffrey Snyder ${ }^{[\mathrm{j}]}$, Janine George ${ }^{[\mathrm{k}][1]}$ Matthias T. Agne ${ }^{*[\mathrm{~m}]}$, Wolfgang G. Zeier*[a][m]

${ }^{a}$ Institute of Inorganic and Analytical Chemistry, University of Münster, D-48149 Münster, Germany

${ }^{b}$ George W. Woodruff School of Mechanical Engineering, Georgia Institute of Technology, Atlanta, Georgia 30332, United States

${ }^{c}$ Institute of Physical Chemistry, University of Münster, D-48149 Münster, Germany

${ }^{d}$ International Graduate School for Battery Chemistry, Characterization, Analysis, Recycling and Application (BACCARA), University of Münster, D-48149 Münster, Germany

${ }^{e}$ Global Zero Emission Research Center, National Institute of Advanced Industrial Science and Technology (AIST), Tsukuba, Ibaraki 305-8569, Japan

${ }^{f}$ Interdisciplinary Materials Research Center, School of Materials Science and Engineering, Tongji University, 201804 Shanghai, China

${ }^{g}$ Institute of Materials Physics, University of Münster, D-48149 Münster, Germany

${ }^{h}$ Institute of Condensed Matter and Nanoscience, Université Catholique de Louvain, 1348 Ottignies-Louvain-la-Neuve, Belgium

${ }^{i}$ Dartmouth College, Thayer School of Engineering, New Hampshire, 03755 Hanover, United States

${ }^{j}$ Department of Materials Science and Engineering, Northwestern University, Illinois, 60208 Evanston, United States

${ }^{k}$ Federal Institute for Materials Research and Testing (BAM), D-12205 Berlin, Germany

Institute of Condensed Matter, Theory and Solid-State Optics, Friedrich Schiller University, 07743 Jena, Germany

${ }^{m}$ Institut für Energie- und Klimaforschung (IEK), IEK-12: Helmholtz-Institut Münster, Forschungszentrum Jülich, 48149 Münster, Germany

Corresponding author emails: m.agne@fz-juelich.de,wzeier@uni-muenster.de 
Ultra-low lattice thermal conductivity as often found in superionic compounds is greatly beneficial for thermoelectric performance, however, a high ionic conductivity can lead to device degradation. Conversely, high ionic conductivities are searched for materials in solid-state battery applications. It is commonly thought that ionic transport induces low thermal conductivity and that ion and thermal transport are not completely independent properties of a material. However, no direct comparison or underlying physical relationship has been shown between the two. Here we establish that ionic transport can be varied independent of thermal transport in $\mathrm{Ag}^{+}$superionic conductors, even though both phenomena arise from atomic vibrations. Thermal conductivity measurements, in conjunction with two-channel lattice dynamics modeling, reveals that the vast majority of $\mathrm{Ag}^{+}$vibrations have non-propagating diffuson-like character, which provides a rational for how these two transport properties can be independent. Our results provide conceptually novel lattice dynamical insights to ionic transport and confirm that ion transport is not a requirement for ultra-low thermal conductivity. Consequently, this work bridges the fields of solid state ionics and thermal transport, thus providing design strategies for functional ionic conducting materials from a vibrational perspective.

\section{Introduction}

Superionic conductors are sparking tremendous interest in multiple fields. While fast ionic conductors are searched for solid state batteries, ${ }^{1}$ they also often possess low lattice thermal conductivities essential for high thermoelectric efficiencies. ${ }^{2-4}$ In fact, many $\mathrm{Ag}^{+}$and $\mathrm{Cu}^{+}$ superionic thermoelectric materials have thermal conductivities below the theoretical "minimum" thermal conductivity for solids, leading some to the suggestion that ion mobility makes these materials more liquid-like. ${ }^{2,4}$ However, no physical connections between thermal and ionic transport have thus far been shown. Recently, a foundational misconception within the concept of "phonon liquid electron crystal"2 was proven by showing that transverse phonon 
modes, which should not exist in a liquid, do persist above superionic phase transitions in solids. ${ }^{5,6}$ Concurrently, a universal theory for heat conduction in solids has been developed that suggests low thermal conductivities can arise solely from (static) atomic disorder, strong anharmonicity and/or complex unit cells, all of which are inherent to fast ion conducting materials. ${ }^{3,5,6}$ Lacking a direct comparison between ionic transport data and the thermal and vibrational properties in these systems, the interdependence of both transport processes remains elusive.

Fundamentally, thermal and ionic transport are related at the vibrational level as they both arise from fluctuations of the phonon occupation number $n_{i}$, which is the instantaneous number of vibrations (phonon quanta) that are in a vibrational mode having frequency $v_{i} \cdot{ }^{7-9}$ Thermal transport results from the thermodynamic drive to have a constant phonon occupation (thermal energy) throughout the entire material. Ion transport occurs when a thermal fluctuation is energetically capable of moving the ion between adjacent lattice sites.

The rate at which phonons move throughout a material depends on the character of the phonon modes. Historically, heat transport by phonons was thought to occur in a propagating manner on a length-scale much larger than interatomic distances (phonon-gas model). In disordered, anharmonic and structurally more complex solids, heat transport can alternatively be conducted by fundamentally different transport mechanisms, one of which is the diffuson. ${ }^{10,11}$ In contrast to the phonon-gas model, diffusons correspond to heat transport via local (atomic) scale random walk. ${ }^{12,13}$ This diffusive walk of heat ${ }^{14}$ means that non-propagating vibrations in a solid transfer thermal energy between adjacent diffuson modes, ${ }^{11,12}$ at a much smaller length scale compared to typical propagating modes (shown schematically in Figure $1 \mathrm{a}, \mathrm{b}$ ).

Regardless of the character of the vibrational modes, fluctuations in vibrational energy remain the fundamental origin of thermal transport. ${ }^{7}$ On the other hand, ionic transport is restricted by an activation barrier (nominally referred to as $E_{\mathrm{A}}$ ) that is largely determined by the potential 
energy landscape, i.e. the coordination environment of the mobile species (Figure $1 \mathrm{c}$ ). ${ }^{15}$ With that, the magnitude of the fluctuation needed to move an ion is significantly increased, relative to thermal transport, since the kinetic energy of the ion has to surpass this activation barrier. Despite this conceptual difference there are strong underlying physical relationships suggesting a connection between thermal diffusons and ionic diffusion: 1) fast ionic transport is achieved in strongly disordered materials ${ }^{16,17}$ and strong disorder promotes the prevalence of thermal transport via diffusons. ${ }^{12}$ 2) Both transport phenomena operate within an atomic-scale random walk, in which local vibrations carry and transfer heat energy or momentum of ions (Figure 1 b, d). ${ }^{12,18}$ 3) Anharmonic lattice vibrations enhance diffuson-mediated transport ${ }^{14}$ and, although the characteristic vibration of a mobile ion is usually shown using a harmonic potential well, an ion jump is an intrinsically anharmonic process. ${ }^{19}$

Motivated by these apparent similarities, this work aims to provide a stepping stone in unifying the concepts of thermal and ionic transport by experimentally accessing both processes and answering the following questions: 1) Are significant diffuson contributions to thermal conductivity present in superionic conductors? 2) Which vibrational modes characterize both transport processes, and 3) how does the magnitude of ion transport influence thermal transport? Especially the latter is of significant relevance, with high ionic conductivities hindering the long-term stability of superionic conductors in thermoelectric devices ${ }^{20}$ and with batteries comprised of ionic conductors needing thermal management to decrease charging times and improve safety. ${ }^{1}$ 
a

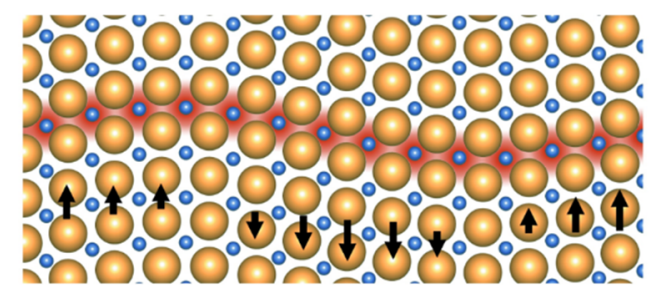

C Energy landscape of ion jumps

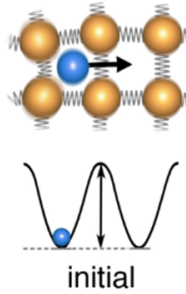

Phonon gas model

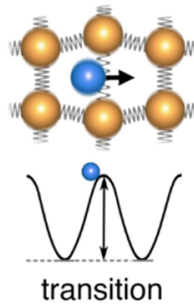

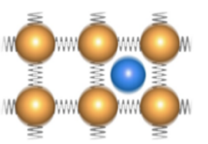

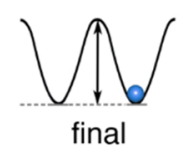

d

Ionic random walk

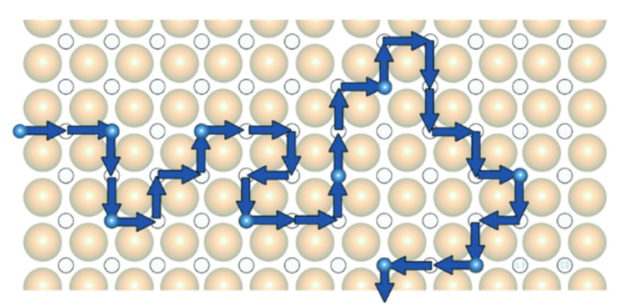

Figure 1: Schematic representation of phonon, diffuson and ion transport. (a) A long-range phonon mode, with highly correlated atomic displacements (black arrows), carriers heat (in red) throughout the lattice in a manner analogous to a gas (i.e. the phonon gas model). (b) Diffusons, which do not tend to have highly correlated atomic displacements (black arrows), transfer heat (in red) on a local scale as a random walk, similar to ionic motion. (c) Schematic of an ion jump and corresponding energy landscape that has a notable barrier to transport. (d) Ionic diffusion as governed by a random walk (portion of random walk shown).

\section{Results and Discussion}

Structural features. To answer these questions, a successful isovalent substitution from $\mathrm{Ag}_{8} \mathrm{SiSe}_{6}$ to $\mathrm{Ag}_{8} \mathrm{GeSe}_{6}$ and $\mathrm{Ag}_{8} \mathrm{SnSe}_{6}$ is achieved as shown by the linear increase of the lattice volume (Supplemental Note 1,2$){ }^{21}$ These materials are superionic conductors and have been intensely investigated for their thermoelectric transport properties due to the low thermal conductivity. ${ }^{3,22,23}$ The different compositions have varying room-temperature structures, ${ }^{24,25}$ but ultimately undergo a phase transition into the same cubic structure that is characterized by its strongly disordered $\mathrm{Ag}^{+}$sublattice (Figure 2 a and Supplemental Note 2). ${ }^{3,22}$ Structurally, there are a large number of $\mathrm{Ag}^{+}$sites that are tetrahedrally coordinated and have face-sharing 
connectivity. These sites have an average occupation of only $25 \%$. While the low-temperature phase of $\mathrm{Ag}_{8} \mathrm{SiSe}_{6}$ is cubic with an ordered $\mathrm{Ag}^{+}$sublattice, ${ }^{24}$ both $\mathrm{Ag}_{8} \mathrm{GeSe}_{6}$ and $\mathrm{Ag}_{8} \mathrm{SnSe}_{6}$ crystallize in an orthorhombic structure at room-temperature (Supplemental Note 2). In the orthorhombic phase, the $\mathrm{Ag}^{+}$sublattice consists of five distinct lattice sites, each either tetrahedrally or trigonally coordinated by $\mathrm{Se}^{2-}$ arranged in a corner- or edge-sharing fashion, respectively. Near the phase transition, the lattice volume increases strongly in a small temperature window before it settles into a volumetric thermal expansion coefficient ranging from 7.7 to $8.7 \times 10^{-5} \mathrm{~K}^{-1}$, with an average of $8.2 \pm 0.5 \times 10^{-5} \mathrm{~K}^{-1}$ for all compositions (Supplemental Note 2, 3).

While the influence of local structural changes are usually employed as an important metric in the understanding of ionic conduction ${ }^{26}$, the underlying vibrational influences remain elusive. Here, we focus on the vibrational characterization of these superionic conductors to give new insights relating vibrations, ionic jumps and thermal transport.

Vibrational frequencies. The vibrational spectrum of a material has significant importance for the fields of ionic conduction and thermal conduction. For ionic transport, the attempt frequency of the mobile species is included in the Arrhenius pre-factor (Supplemental Note 4). ${ }^{15,27}$ Often the Debye frequency $v_{\mathrm{D}}$ is used to approximate the attempt frequency despite not probing the vibrations of the mobile species directly. In fact, especially in disordered and soft materials, the Debye-frequency often fails its original purpose to estimate the maximum vibrationalfrequency, but rather falls into the center of the vibrational density of states. ${ }^{12,28}$ With that, it provides a useful experimental insight to the average dynamics of the lattice, but it should not be expected to be an accurate descriptor for the vibrational properties of the mobile ion. ${ }^{28} \mathrm{~A}$ better descriptor for the attempt frequency would be a vibrational frequency $v_{\mathrm{E}}$ specific for the mobile ion that is conceptually similar to an Einstein oscillator. ${ }^{29,30}$ This concept is especially useful for materials with an underlying guest-host structural motif, like the mobile ion within a 
rigid sub-lattice in superionic conductors ${ }^{2}$ or guest atoms and associated "rattler modes" in the skutterudites and clathrates, which have rather dispersion-less phonon branches (Supplemental Note 5$).{ }^{31}$
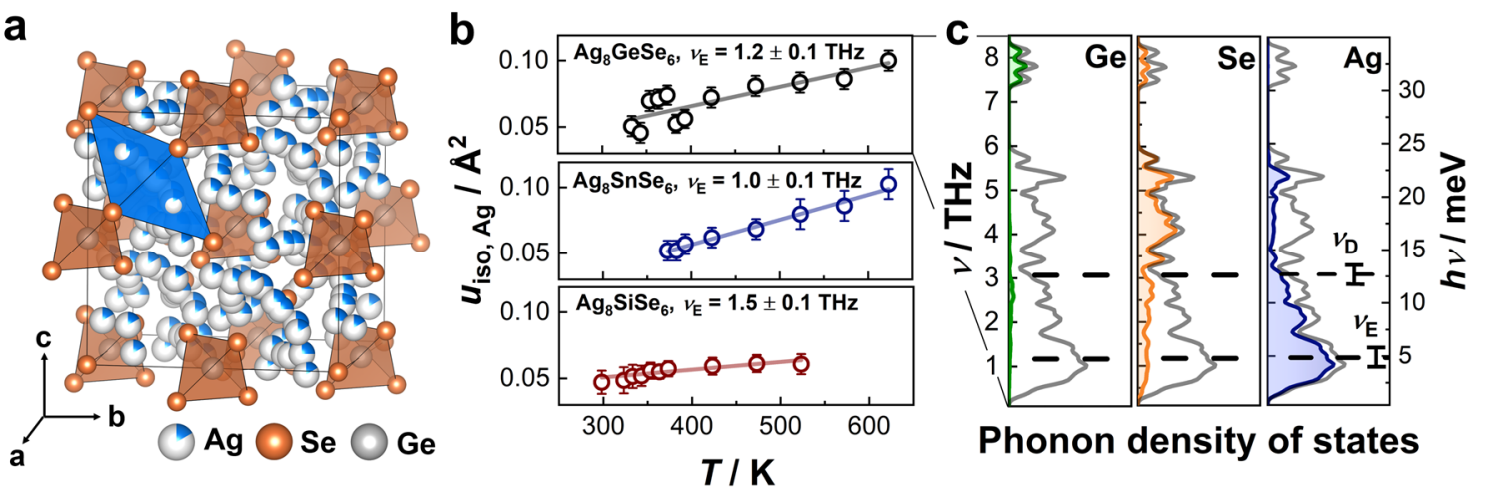

Figure 2: Structural and vibrational characterization of $\mathrm{Ag}^{+}$argyrodites. (a) Structural view of the cubic $\mathrm{Ag}_{8} \mathrm{GeSe}_{6}$, showing the anionic framework (i.e. Ge-centered Se tetrahedra, orange in figure), a representative $\mathrm{Ag}^{+}$polyhedral pathway (blue polyhedral) and strong $\mathrm{Ag}^{+}$disorder (represented by low fractional occupancy, i.e. partially blue $\mathrm{Ag}^{+}$sites) (b) An increase in the thermal displacement parameter of $\mathrm{Ag}^{+}$is observed with increasing temperature, corresponding to Einstein frequencies in the range of $v_{E}=1.0 \pm 0.1 \mathrm{THz}$ to $v_{E}=1.5 \pm 0.1 \mathrm{THz}$. (c) Atom-projected phonon density of states of the orthorhombic phase of $\mathrm{Ag}_{8} \mathrm{GeSe}_{6}$ showing the agreement of the Einstein-like $\mathrm{Ag}^{+}$vibrations with the low-frequency peak of the $\mathrm{Ag}^{+}-$ projected DOS. The Debye frequencies reflect the center of the calculated phonon DOS.

Here, we find that the average $\mathrm{Ag}^{+}$vibrational frequency $(1.2 \pm 0.2 \mathrm{THz}$; range $=1.0-1.5 \mathrm{THz})$ determined from X-ray diffraction measurements is consistent with the average $\mathrm{Ag}^{+}$frequency found computationally $(2 \mathrm{THz}$ ), and perfectly aligns with the lowest frequency peak in the vibrational density of states (Figure 2 c). Meanwhile, the average Debye frequency $(3.1 \pm 0.2$ $\mathrm{THz}$; range $=2.5-3.4 \mathrm{THz}$ ) found from speed of sound measurements does capture the average vibrational frequency of the entire density of states $(\sim 3 \mathrm{THz})$, but does not correspond to any particular feature. A detailed discussion of the measurements and further comparison can be 
found in the Supporting Information (Supplemental Notes 2,3,5). It is important to note that the ability to directly probe vibrations of the mobile ion provides experimental insights to the frequency spectrum relevant for ionic transport. From a thermal transport point-of-view, the Debye frequency is frequently shown to provide a strong and simple approximation even for complex materials, ${ }^{32-34}$ while the determination of Einstein frequencies can provide a useful tool if atom-specific frequencies play a significant role. ${ }^{31,35}$ Thus, the characterization of both $v_{\mathrm{D}}$ and $v_{\mathrm{E}}$ provides complementary information in examining thermal and ionic transport together.
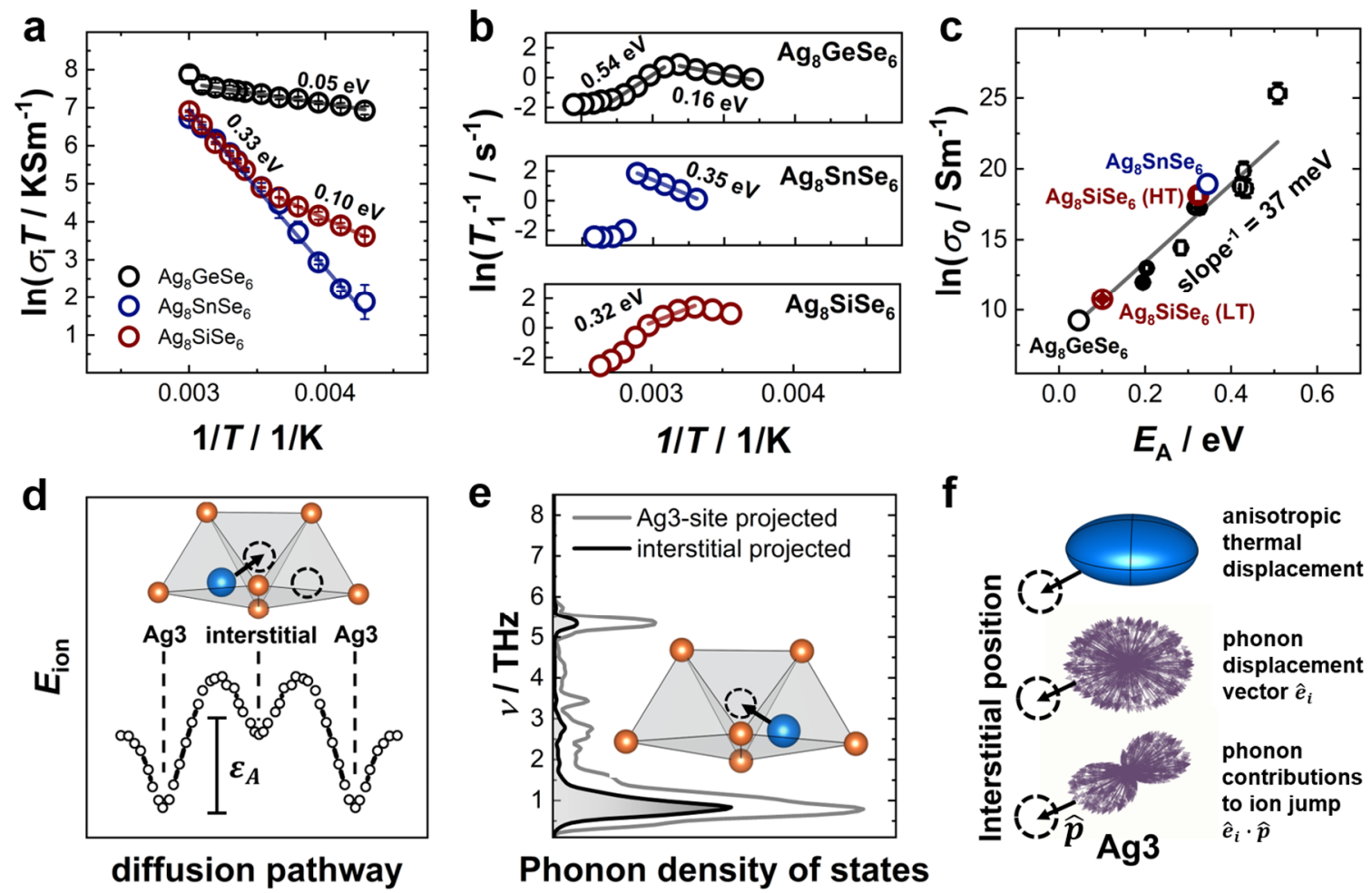

Figure 3: Ionic transport phenomena at macroscopic and atomic scales. (a) Arrhenius temperature dependence of ionic transport, where the slope is defined by the activation barrier $E_{\mathrm{A}}$, as obtained from impedance spectroscopy measurements of the ionic conductivity $\sigma_{\mathrm{i}} .(b)$ Arrhenius plot of the ${ }^{109} \mathrm{Ag} T_{1}$ relaxation times determined from nuclear magnetic resonance spectroscopy, corroborating the magnitudes of $E_{\mathrm{A}}$ found from impedance spectroscopy. (c) The relation between the pre-factors $\sigma_{0}$ and activation energies $E_{\mathrm{A}}$ of ionic conductivity is observed 
to follow Meyer-Neldel behavior (shown as a linear correlation in this plot whose slope has units of inverse energy), which can be expected when composition does not drastically affect the vibrational spectrum. (d) Schematic representation of an exemplary diffusion pathway (Ag3-Ag3) and the changing potential energy of the ion (estimated from bond valence sums) as well as the corresponding local structural changes (inset). (e) Theoretical evaluation of the jump from a vibrational point of view. Projecting the site-specific density of states towards the interstitial position indicates that low-frequency vibrations are particularly significant for this ion jump. (f) Comparison of the thermal displacement ellipsoid and the eigenvector-distribution of the Ag3 site, calculated by density functional theory. Additionally, the distribution of phonon displacement vectors $\hat{e}_{i}$ with contributions towards the interstitial position (defined by the unit vector $\hat{p}$ ) shows alignment with the preferred orientation of the displacement ellipsoid. With that, the $\mathrm{Ag}^{+}$on this lattice site has an inherently higher displacement in the direction of the jump, typically believed to be favorable for ionic transport.

$\mathrm{Ag}^{+}$ionic transport. The thermoelectric properties of these fast ionic conductors, such as ultralow thermal conductivity, are often inferred to be connected to ion mobility, but the corresponding magnitude of ionic conductivity is rarely (if ever) reported. Here, we evaluate the magnitude and temperature dependence of the ion conduction of $\mathrm{Ag}_{8}(\mathrm{Si}, \mathrm{Ge}, \mathrm{Sn}) \mathrm{Se}_{6}$ solid solutions (see Supplemental Note 4).

The ionic conductivities vary strongly upon substitution with room-temperature values ranging from 0.088 to $5.0 \mathrm{~S} / \mathrm{m}$ as determined by impedance spectroscopy. The respective Arrhenius behavior for $\mathrm{Ag}_{8} \mathrm{SiSe}_{6}, \mathrm{Ag}_{8} \mathrm{GeSe}_{6}$ and $\mathrm{Ag}_{8} \mathrm{SnSe}_{6}$ reveals significant changes to the activation barrier and, with that, the temperature dependence of ionic transport (Figure 3 a). Here, $\mathrm{Ag}_{8} \mathrm{GeSe}_{6}$ exhibits the lowest activation barrier of $0.05 \mathrm{eV}$ while both $\mathrm{Ag}_{8} \mathrm{SiSe}_{6}$ and $\mathrm{Ag}_{8} \mathrm{SnSe}_{6}$ show larger barriers of $\sim 0.3 \mathrm{eV}$ at temperatures above $298 \mathrm{~K}$. These results are confirmed by nuclear magnetic resonance spectroscopy (Figure $3 \mathrm{~b}$ ), which indicates that the activation 
energies are predominantly a bulk property of the solid and not caused by microstructural differences. Furthermore, not only the disordered high-temperature cubic phase but also the ordered low-temperature phases of the $\mathrm{Ag}^{+}$argyrodites have significant ionic conduction.

For all investigated materials, the logarithm of the pre-exponential factor scales linearly with the activation barrier (Figure $3 \mathrm{c}$ ). This is a common occurrence in ionic materials, known as the Meyer-Neldel rule, ${ }^{36-38}$ and is attributed to the interrelation of migration enthalpy (i.e. $\left.\Delta H_{\mathrm{m}} \propto E_{\mathrm{A}}\right)$ and entropy (i.e. $\Delta S \propto \ln \left(\sigma_{0}\right)$ ). This relation has been derived before using transition state theory and the multi-excitation entropy model. ${ }^{36,37}$ Specifically, it is believed that the inverse slope of the Meyer-Neldel plot is related to the energy of the vibrational modes that participate in ionic conduction, as well as a characteristic number of vibrations. However, a consistent definition of these quantities has been lacking. ${ }^{37}$ Nevertheless, an inverse slope of $\sim 37 \mathrm{meV}$ is in agreement with those reported for other superionic conductors. ${ }^{39}$ In comparison to the energy range of the calculated phonon density of states it is unclear what this value really represents, except that the vibrational states of the $\mathrm{Ag}^{+}$ions do not change significantly through the substitution series, and that the overall vibrational properties are not significantly affected by the composition. By novel considerations of the phonon entropy (Supplemental Note 6), we show that using an average frequency of $\mathrm{Ag}^{+}$vibrational modes $(\sim 2 \mathrm{THz})$ in the evaluation of the Meyer-Neldel slope coincides with a characteristic number of vibrations ( 4.6 phonons) nearby the value expected ( 3.1 phonons) from Bose-Einstein statistics at room temperature. This result provides confidence that it is, in fact, the $\mathrm{Ag}^{+}$vibrations that are responsible for ion transport within the multi-excitation model.

So far, we have used $E_{\mathrm{A}}$ and $v_{\mathrm{E}}$ as bulk isotropic properties of the solid, but it is important to remember the local scale on which each ion jump occurs. Consequently, there are local activation barriers $\varepsilon_{\mathrm{A}}$ for each possible jump from a given $\mathrm{Ag}^{+}$site, and each $\mathrm{Ag}^{+}$site can have its own vibrational spectrum. Here, we examine one exemplary $\mathrm{Ag}^{+}$jump from both a static 
(bond valence sum analysis) and lattice dynamical view-point (see Supplemental Note 7 for further discussion). The bond valence sum analysis reveals the potential energy landscape of an ionic jump between two tetrahedrally coordinated $\mathrm{Ag} 3$ equilibrium sites (Figure $3 \mathrm{~d}$ ). The $\mathrm{Ag}^{+}$ ion has to jump through the face of the tetrahedron (local potential energy maximum) to a tetrahedrally coordinated interstitial site (local potential energy minimum) from which it can jump to either adjacent $\mathrm{Ag}^{+}$site. The local activation barrier for this jump is expected to be small, since only minor coordination changes (from $4-3$ - 4) are necessary and the interstitial site is energetically stablilized. ${ }^{40}$

From a lattice dynamics perspective, it is possible to reduce the partial $\mathrm{Ag}^{+}$vibrational density of states (blue shading in Figure 2 b) to a site-specific density of states (Figure 3 e). From here, one can identify which frequencies are likely important for different jumps by weighting vibrational modes according to their projection in the jump direction (Figure $3 \mathrm{e}$, f, Supplemental Note 7). The average frequency in the direction of the jump (1.4 THz) is lower compared to both, the site- $(1.7 \mathrm{THz})$ and atom-projected case $(2.0 \mathrm{THz})$. The lower average frequency implies weaker bonding in the jump direction, and thus a lower potential barrier from a lattice dynamical viewpoint, confirming the results from bond valence sum analysis. Lower frequencies also imply a larger amplitude of vibration in the direction of the jump, which should manifest itself in experimentally accessible anisotropic thermal displacement parameters (shown schematically as a blue ellipsoid in Figure 3 f). Here, the calculated anisotropic displacement originates from the distribution of the phonon displacement vectors, the directional preference of which are in good alignment with the jump direction in this example (purple arrows in Figure $3 \mathrm{f}$ ).

Typically, changes in the activation barrier and magnitude of ionic conductivity are discussed from the static perspective, e.g., electronegativity, unit cell and pathway volumes. ${ }^{15}$ The sitespecific and directional analysis of phonon modes proposed here allows for identification of 
prominent vibrational frequencies of the mobile ion from lattice dynamics calculations. The importance of tracking specific vibrational modes within molecular dynamics simulations was recently discussed by Gordiz et al. ${ }^{41}$ They showed that the vibrational contributions to ionic conduction are heavily frequency-dependent. Herein, we also assess the relevance and vibrational character (phonon-gas-like or diffuson-like) of phonon modes for thermal transport. With that, we aim to draw conclusions about the interdependence of both phenomena.

Diffuson-mediated thermal transport. The ultra-low thermal conductivities of superionic conductors are often attributed to (static and dynamic) atomic disorder, soft bonding, lowfrequency optical phonon modes, anharmonicity and complex crystal structure. ${ }^{42}$ All of these parameters are associated with either lowering the phonon group-velocity or increasing the phonon scattering rate, in context of the phonon-gas model. ${ }^{3,22,23}$ While these structural and vibrational features are likely to factor into the characteristics of thermal transport, the vicinity to the Ioffe-Regel limit and the associated concept of minimum lattice thermal conductivity suggests the phonon-gas model is likely an incomplete description of thermal transport in these systems. $^{12}$ 

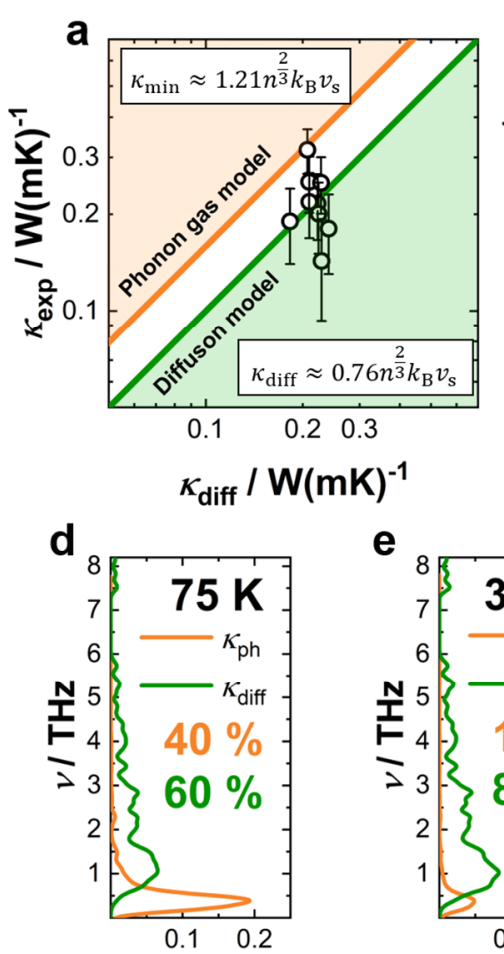

$\kappa(v) / \mathrm{W}(\mathrm{mKTHz})^{-1} \kappa(v) / \mathrm{W}(\mathrm{mKTHz})^{-1}$

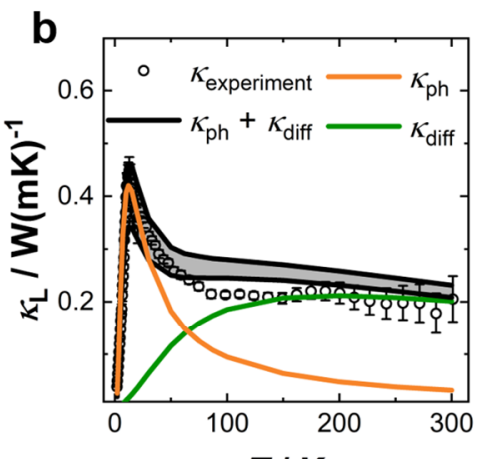

$T / K$

f
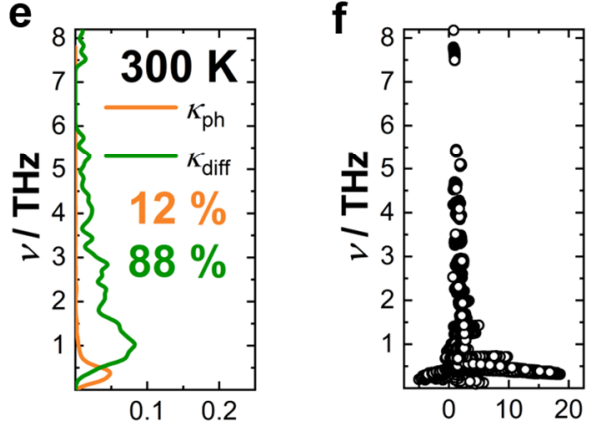

$\gamma(v)$

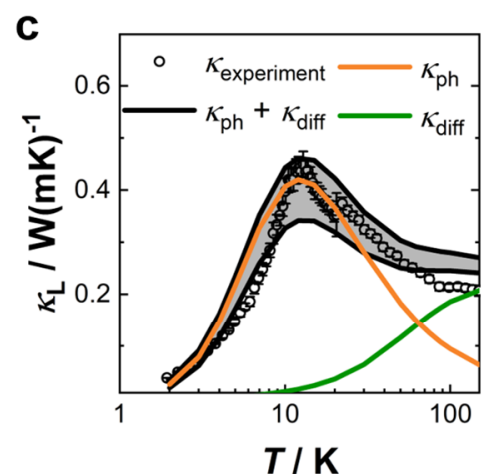

g

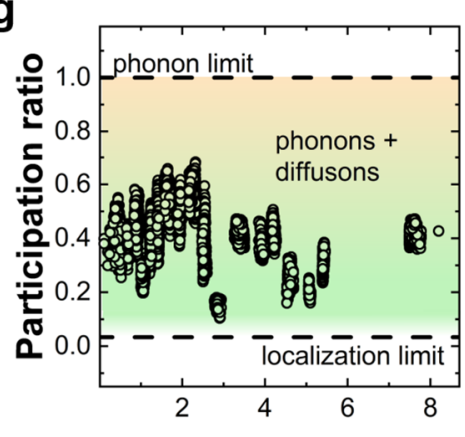

$v / \mathrm{THz}$

Figure 4: Vibrational character and thermal transport in $\mathrm{Ag}_{8} \mathrm{GeSe}_{6 .}$ (a) The high-temperature lattice thermal conductivity of all compositions at $475 \mathrm{~K}$ in comparison to the Cahill (phonongas minimum) and diffuson limits. The phonon-gas model is insufficient in describing the data, with experimental results consistently being underneath the theoretical minimum. (b) Lattice thermal conductivity and corresponding two-channel lattice dynamical transport model from 2 to $300 \mathrm{~K}$ which shows the transition from phonon gas-like conductivity (below $100 \mathrm{~K}$ ) to diffuson dominated thermal transport (above $100 \mathrm{~K}$ ). Here, the two lines of the total thermal conductivity model represent the upper and lower limit stemming from a slight transport anisotropy. (c) A close-up of the low-temperature behavior emphasizes the good fit of the phonon peak. The spectral thermal conductivity of phonon- and diffuson-like modes are shown at (d) $75 \mathrm{~K}$ and (e) $300 \mathrm{~K}$, both showing the fast decline of phonon-character upon increasing temperature and large diffuson-contributions of the $\mathrm{Ag}^{+}$-dominated modes (below $\sim 2.4 \mathrm{THz}$ ).

(f) The spectral Grüneisen parameter shows large anharmonicities below 1 THz matching the spectral phonon-contributions, leading to the fast decline of phonon-like transport within the 
same frequency range. $(g)$ The participation ratios of vibrational modes (calculated from the mode eigenvectors) have values well below the phonon limit for spatially extended modes (dashed line, participation-ratio $=1)$ and comparable to amorphous materials ${ }^{9}$ indicating diffuson-like transport complementary to the two-channel modelling.

The measured high temperature lattice thermal conductivities (Supplemental Note 8) show a flat temperature dependence typical for diffuson-mediated transport, independent of the composition, with average values of $0.21 \pm 0.05 \mathrm{~W} / \mathrm{mK}$ at $475 \mathrm{~K}$ (range $0.15 \mathrm{~W} / \mathrm{mK}$ to 0.32 $\mathrm{W} / \mathrm{mK})$. These values are consistently below the theoretical "minimum" thermal conductivity expected from the phonon-gas model and in good alignment with the estimations of a diffuson model (Figure 4 a). ${ }^{12,43}$ To confirm the diffuson-like nature of thermal transport at high temperature, low-temperature thermal conductivities were measured. Here, the lattice thermal conductivity of $\mathrm{Ag}_{8} \mathrm{GeSe}_{6}$ shows that the flat-temperature dependence persists down to $100 \mathrm{~K}$ before a phonon-peak arises around $12 \mathrm{~K}$ (Figure $4 \mathrm{~b}$ ). This exceptional temperature dependence was confirmed by corresponding low-temperature measurements of $\mathrm{Ag}_{8} \mathrm{SiSe}_{6}$ and $\mathrm{Ag}_{8} \mathrm{SnSe}_{6}$ (see Supplemental Note 8).

Two-channel modeling based on the calculated lattice dynamics for $\mathrm{Ag}_{8} \mathrm{GeSe}_{6}$ is utilized to explain these results. Here, the heat current operator matrix is analyzed regarding its diagonal (phonon-like) and off-diagonal (diffuson-like) elements such that the total lattice thermal conductivity of the two-channel model is $\kappa_{\mathrm{L}}=\kappa_{\mathrm{ph}}+\kappa_{\text {diff }}{ }^{13}$ Given the computational cost of third and fourth order force constants, an analytical scattering model was implemented to describe the temperature dependence of the phonon lifetimes (details in Supplemental Note 9). ${ }^{13}$ The analytical model considers phonon-phonon scattering $\left(\tau_{\mathrm{p}}^{-1}\right)$ and boundary scattering $\left(\tau_{\mathrm{b}}^{-1}\right)$ resulting in a total scattering rate of 


$$
\tau^{-1}=\tau_{\mathrm{p}}^{-1}+\tau_{\mathrm{b}}^{-1}=C_{1} \omega^{2} T \cdot e^{-\frac{C_{2}}{T}}+A \omega
$$

with $C_{1}, C_{2}$ and $A$ as constants that describe the experimental data.

The phonon gas channel contributions, $\kappa_{\mathrm{ph}}$, dominate the total lattice thermal conductivity at low-temperatures and well-describe the peak in thermal conductivity (Figure $4 \mathrm{c}$ ). With increasing temperature, the phonon gas channel declines while vice-versa the diffuson contributions, $\kappa_{\text {diff }}$, increase strongly, before plateauing above $100 \mathrm{~K}$. The resulting total lattice thermal conductivity of the two-channel model, $\kappa_{\mathrm{L}}=\kappa_{\mathrm{ph}}+\kappa_{\mathrm{diff}}$, captures both the magnitude and temperature-dependence of the experimental data, not only in the phonon $(T<50 \mathrm{~K})$ and diffuson $(T>100 \mathrm{~K})$ mediated regimes but also at temperatures where both channels have similar contributions. The two lines shown for the total lattice thermal conductivity represent the upper- and lower-limit of the calculation, stemming from a slight anisotropy of the orthorhombic structure (Figure 4 b, c, for detail see Supplemental Note 9). This observation is supported by the reported single-crystal thermal conductivity analysis of $\mathrm{Ag}_{8} \mathrm{SnSe}_{6}$, which found a comparable anisotropy in thermal conduction at $300 \mathrm{~K} .{ }^{44}$ It should be emphasized that the conclusion that the diffuson channel dominates is independent of the form of the analytical scattering functions. Corresponding results utilizing a simplified phonon-phonon scattering term are shown in the Supporting Information (Supplemental Note 9).

The two-channel model additionally allows for a spectral analysis of the thermal conductivity of both channels (Figure $4 \mathrm{~d}$, e). The contributions from the phonon gas channel arise from the lowest frequency modes below $1 \mathrm{THz}$, while the spectral thermal conductivity of the diffusonchannel is extended over the whole frequency range. Note that the spectral thermal conductivity is not expected to be zero at zero frequency but is an apparent artifact of uniform q-meshing. ${ }^{45}$ Here, the minimal contributions of the high-frequency vibrations, dominated by $\mathrm{M}=\mathrm{Si}, \mathrm{Ge}$ and Sn (see Figure 2 c), explain why no significant changes to the thermal transport are observed in 
the solid-solutions. Nevertheless, at temperatures relevant for ion transport, the $\mathrm{Ag}^{+}$vibrations have predominantly diffuson-like character.

Evaluation of the mode-specific Grüneisen parameters (Supplemental Note 5) lends an explanation for the strong decline of the phonon gas channel (Figure $4 \mathrm{f}$ ). Here, large Grüneisen parameters are found in the same frequency range in which the phonon gas channel contributions are the strongest (Figure $4 \mathrm{~d}$, e). Therefore, the extensive anharmonicity at low frequencies is believed to be the driving factor for the strong suppression of the phonon-channel and the subsequent transition to diffuson-dominated thermal transport. This was captured phenomenologically by our analytical scattering function. It is not surprising that such large Grüneisen parameters exist since anharmonic vibrations have been linked to ionic transport. ${ }^{5,6,46}$ Having assessed the vibrational character of phonon modes from thermal transport behavior, it is also possible to characterize the vibrational character directly from the mode eigenvectors. The spatial extension and "shape" of phonon modes has been used previously to distinguish vibrational characters. Here, we use the participation ratio for such analysis (Figure $4 \mathrm{~g}$, details in Supplemental Note 10). ${ }^{9,10}$ A participation ratio close to unity is indicative of a high spatial extension of a mode and typically found in simple crystalline materials like c-Si that have textbook phonon-gas transport, ${ }^{10}$ and so a participation ratio of 1 can be called the phonon limit. Participation ratios below $\sim 0.1$ are typical for localized modes that do not contribute to thermal transport, and the localization limit is, by definition, when only 1 atom (out of all the atoms in the unit cell) participates in the vibrational mode. ${ }^{9}$ The calculated participation ratios are in the intermediate range, comparable to those of amorphous materials like a-C and a-SiO${ }_{2}{ }^{9}$, despite the crystalline nature of $\mathrm{Ag}_{8} \mathrm{GeSe}_{6}$. While the participation ratio cannot clearly separate phonons and diffusons, ${ }^{9,47}$ the similarity to the results of amorphous materials strongly indicates the presence of diffuson-like vibrations complementary to the two-channel model. Again, this indicates that $\mathrm{Ag}^{+}$vibrations in particular are predominantly diffuson-like. 
These results confirm diffuson-mediated thermal transport in the $\mathrm{Ag}^{+}$argyrodites at temperatures relevant for thermoelectrics and ionic conduction. Accordingly, the phonon-gas channel has only a minor contribution. It can be concluded that a complex crystal structure, resulting in many energetically close phonon modes, and relatively large anharmonicities are sufficient to achieve diffuson-dominated thermal transport in crystalline materials. This is to say that we do not need to explicitly invoke ion transport effects in order to capture the magnitude of thermal conductivity. Thus, significant diffuson-contributions can be expected in other structurally well-defined materials.

Connecting ionic and thermal diffusion. The results presented above have the surprising observation that the thermal conductivity of $\mathrm{Ag}^{+}$argyrodites is seemingly independent of ionic transport as the diffuson-based thermal conductivity does not change significantly, while the changes in ion transport (based on Nernst-Einstein diffusion coefficients, Supplemental Note 4) are large across the same temperature range (Figure 5 a). To comprehend the underlying differences of thermal and ionic transport one can examine the number of $\mathrm{Ag}^{+}$ions participating in the respective processes. While all $\mathrm{Ag}^{+}$vibrational modes (and with that all $\mathrm{Ag}^{+}$ions) contribute to thermal transport at high temperatures (e.g., Figure 4 e), the instantaneous fraction of mobile $\mathrm{Ag}^{+}$participating in ionic transport is given by the Boltzmann distribution,

$$
\frac{N_{\text {mobile }}}{N_{\text {total }}}=\exp \left(-\frac{E_{\mathrm{A}}}{k_{\mathrm{B}} T}\right)
$$

where $N_{\text {mobile }}$ is the number density of mobile $\mathrm{Ag}^{+}$ions, $N_{\text {total }}$ is the total number density of $\mathrm{Ag}^{+}$ions, and $E_{A}$ is the macroscopic activation barrier (Figure $5 \mathrm{~b}$ ). Consequently, even with an exceptionally low activation barrier of $0.05 \mathrm{eV}$, comparable to other superionic conductors like $\mathrm{Cu}_{2} \mathrm{Se}^{48}$ only $\sim 10 \%$ of the ions are thermally mobile (without an applied electric field) at any given time over the investigated temperature range. For higher activation barriers, this fraction stays well below $1 \%$. This observation sheds light on the misconception of a "liquid-like" sublattice, given that temperatures of more than $1000 \mathrm{~K}$ would be necessary to even reach a 
$50 \%$ thermally mobile sublattice. While instantaneous mobile ion fractions on the order of several percent could be expected to significantly impact thermal transport within the phonon gas model, the nature of diffuson modes makes them less likely to be largely affected so long as the mode energies do not change drastically as more ions become mobile. This does not seem to be the case, nor does the magnitude of ionic conductivity suggest any significant thermal transport by ion mobility (see Supplemental Note 11).

At a deeper level, the difference between ionic and thermal transport is better understood by considering their similarities, that is the microscopic origin of both processes: phonon occupation fluctuations. Thermal transport in solids arises from any deviation $\left(n_{i}-n_{i}^{0}\right) \neq 0$ from the equilibrium phonon occupation number $n_{i}^{0}$, with $n_{i}$ being the instantaneous occupation of mode $i$ (see Supplemental Note 6). Only the drift velocity of the phonon (e.g., phonon-gaslike or diffuson-like) determines the magnitude of its contribution to transport. In contrast, ionic motion is an activated process such that a critical fluctuation has to be reached for the ion to overcome the activation barrier. The magnitude of this critical fluctuation will depend on the vibrational mode(s) involved and the specific jump that is considered (for example see Figure $3 \mathrm{~d}$-f). For a single vibrational mode that oscillates in the jump direction, the critical number of phonons $n^{\mathrm{c}}$ required for a jump to occur is

$$
n \geq n^{\mathrm{c}}=\left\lceil\frac{\varepsilon_{\mathrm{A}}}{h v}\right\rceil
$$

where $v$ is the frequency of the phonon mode and $\varepsilon_{\mathrm{A}}$ corresponds to the activation barrier of this particular jump (for a mode-specific description see Supplemental Information 6). The ceiling operator is used because phonons are quantized. Intuitively, larger barriers require larger phonon fluctuations. For a $\mathrm{Ag}^{+}$vibrational mode where $h v \approx 5 \mathrm{meV}$, then $n^{c} \approx 10$ to 100 phonons using macroscopic values of $E_{\mathrm{A}}$ for $\varepsilon_{\mathrm{A}}$ in Eq. 3 . 
Similar to the fraction of mobile ions (Eq. 2), only a relatively small fraction of all phonon occupation fluctuations can result in an ionic jump, even if multiple modes participate in the fluctuation. When calculating the probability that a fluctuation is large enough for ionic transport (see Supplemental Information 6) it is significantly lower (blue area in Figure 5 c) than, for example, the probability that a fluctuation is within one standard deviation of the equilibrium phonon occupation number $n^{0}$ (red area in Figure $5 \mathrm{~b}$ ). Since the standard deviation of an exponential distribution is equal to its average value, it follows that the majority of phonon fluctuations are within the bounds $0 \leq n \leq 2 n^{0}$, and $n^{0} \approx 5.0$ phonons for $\mathrm{Ag}^{+}$vibrational modes near room temperature. From this perspective it is not surprising that thermal transport is independent of the activation barrier or ionic transport (Figure $5 \mathrm{c}$ ) since thermal transport is dominated primarily by average phonon fluctuations.

Discussing ion jumps in the context of phonon fluctuations is a novel concept and may be a first step towards a lattice dynamical theory of ion transport. Generally, one could use the jumpdirection projected density of states for each $\mathrm{Ag}^{+}$site to elucidate jump probabilities for each frequency and each $\varepsilon_{\mathrm{A}}$ (compare Figure $3 \mathrm{~d}$, e). From our frequency-dependent analysis, we reach an important conclusion that lower frequencies are more beneficial for ionic transport (Supplemental Note 6), independent of the material, temperature or activation barrier. This is a crucial insight for ion conduction research showing that the local jump dynamics need to be tailored rather than the global lattice dynamics. ${ }^{27,28}$ The inherently low frequencies of $\mathrm{Ag}^{+}$ vibrations in the $\mathrm{Ag}_{8} \mathrm{MSe}_{6}$ argyrodites may thus be a contributing factor to their superionic behavior.

The prominent contribution of $\mathrm{Ag}^{+}$vibrational modes to both thermal and ionic transport is made clear by comparing the vibrational frequencies of those found experimentally and from the calculated density of states (Figure $5 \mathrm{~d}$ ). First, the average diffuson frequency found from the spectral two-channel calculation $\left(v_{\text {diff }}=2.3 \mathrm{THz}\right)$ is in excellent agreement with the value 
estimated experimentally $\left(v_{\text {diff }}=2.2 \pm 0.2 \mathrm{THz}\right.$, range $1.9 \mathrm{THz}$ to $\left.2.4 \mathrm{THz}\right)$ using an analytical diffuson transport model (Supplemental Note 3). ${ }^{12}$ Comparatively, an average ionic transport frequency is found by using the average of the partial $\mathrm{Ag}^{+}$density of states $\left(v_{\mathrm{Ag}^{+}}=2.0 \mathrm{THz}\right)$. Meanwhile, the Einstein frequency determined from X-ray diffraction experiments $\left(v_{\mathrm{E}}=1.2\right.$ $\mathrm{THz}$ ) coincides with the maximum of the partial $\mathrm{Ag}^{+}$density of states, which consists of modes that contribute to large thermal displacements. The fact that these average frequencies all reside between 1 and $3 \mathrm{THz}$ where $\mathrm{Ag}^{+}$makes up $~ 70 \%$ of the vibrational density of states, and that $\mathrm{Ag}^{+}$vibrations have been shown to be largely diffuson-like in nature means that diffusons are foundational to understanding both thermal and ionic transport in these materials. Consequently, the vibrational character is likely important to understand transport in other ionic conductors, with or without ultralow thermal conductivity.
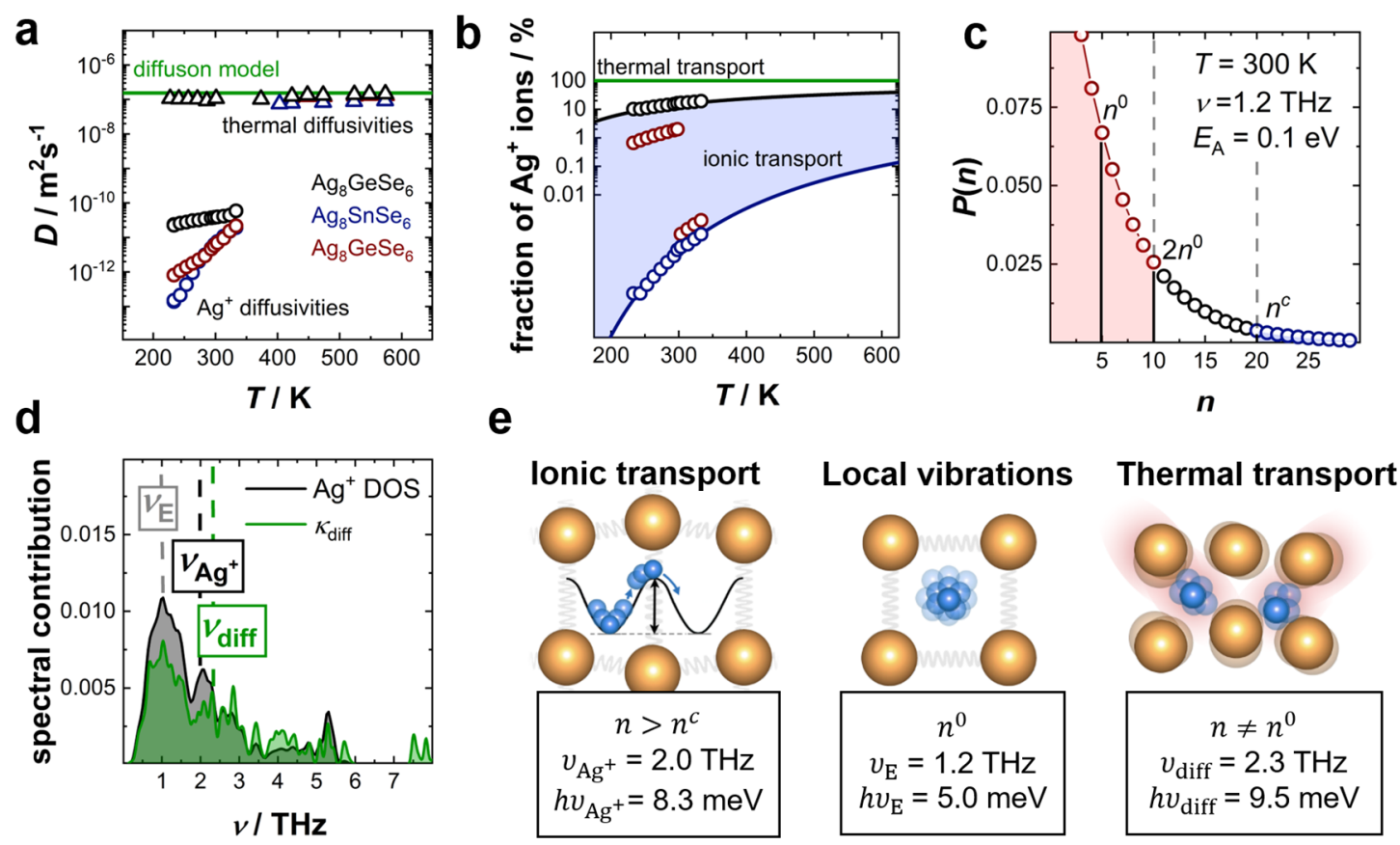

Figure 5. Relations between ionic and thermal transport. (a) Comparing the thermal and ionic diffusivities exemplifies the differences in macroscopic transport behavior, particularly the activated behavior of ionic diffusion has no discernable influence on the temperatureindependent diffuson thermal diffusivity. (b) At any moment in time, all $\mathrm{Ag}^{+}$ions in the lattice 
are expected to participate in thermal transport, but this is not the case for ionic transport. The number of mobile ions is increased with increasing temperature and decreasing activation barrier. Even considering an activation barrier as low as $0.05 \mathrm{eV}\left(\mathrm{Ag}_{8} \mathrm{GeSe} \mathrm{C}_{6}\right)$ only $\sim 10 \%$ of the $\mathrm{Ag}^{+}$ions are thermally mobile (near room temperature) at any instant. (c) The probability distribution function of phonon occupations $P(n)$ gives the instantaneous probability that there are $n$ phonons in a vibrational mode of a given $\mathrm{Ag}^{+}$ion. The red area indicates that typical fluctuations in phonon occupation are within one standard deviation of the equilibrium phonon occupation number $n^{0}$, whereas the blue area indicates there is a much lower probability that there are enough phonons to facilitate ion transport (i.e. $n \geq n^{\mathrm{c}}$, where $n^{\mathrm{c}}$ is the critical number of phonons defined by the activation barrier $E_{\mathrm{A}}$ and the frequency $v$ of the vibrational mode). (d) The $\mathrm{Ag}^{+}$vibrational density of states compared to the diffuson spectral thermal conductivity. It can be seen that the vibrational modes relevant for ionic transport are diffuson-like in character and that the average ionic and diffuson frequencies $\left(v_{\mathrm{Ag}^{+}}\right.$and $v_{\mathrm{diff}}$, respectively) fall within the $\mathrm{Ag}^{+}$dominated part of the density of states near to the determined Einstein frequency $v_{E}$. (e) A schematic representation of how ionic motion, thermal conduction and local vibrational properties are fundamentally connected within the phonon occupation fluctuation picture.

Outlook on engineering transport in ionic conductors. As we have shown, the magnitude of ionic conductivity can be changed independently without altering the thermal transport properties that are governed by diffusons. Therefore, strategies to lower the ionic conductivity, and consequently improve the long-term stability in thermoelectric devices, are not expected to have detrimental effects on the desirable low lattice thermal conductivities. This profound insight opens the possibility to tailor superionic conductors and expand their usage in commercial thermoelectric devices ${ }^{20,49}$ by using the known design principles in superionic conductors. ${ }^{15,40}$ 
In addition, our results lead to an important conclusion for the research of solid ionic conductors that are becoming increasingly relevant for the performance optimization of all solid-state batteries. ${ }^{1,26}$ Here, the Meyer-Neldel behavior is a bottleneck in achieving higher ionic conductivities since a lowering of the activation barrier has detrimental effects on the conductivity pre-factor. ${ }^{15,27,36,39}$ With that, reducing the slope of the Meyer-Neldel plot becomes a main goal. $^{39}$ By deriving the multi-excitation entropy using phonon-fluctuation considerations, we show that the Meyer-Neldel slope can be related to the prominent vibrational modes of ionic conduction and a characteristic number of phonons found to be in the range of equilibrium phonon occupations (Supplemental Note 6). ${ }^{7}$ This leads to a novel concept and design principle in solid-electrolyte research: determining and manipulating phonon occupations (e.g. targeted phonon excitation ${ }^{41}$, through opto-electronic stimulation ${ }^{50}$ ) in conjunction with tailoring the site-specific vibrational frequencies and activation energies.

\section{Conclusion}

This work demonstrates that diffuson-mediated thermal transport is dominant in $\mathrm{Ag}^{+}$argyrodite superionic conductors at temperatures relevant for thermoelectrics and ionic transport. An important consequence of this fact is that ionic conductivity can be varied by orders of magnitude without affecting the thermal conductivity. The similarities of the argyrodite structure with lithium and sodium superionic conductors means that diffusons are likely prevalent in battery materials as well. Thus, by understanding the origins of thermal and ionic transport, in particular via vibrational characteristics and phonon occupations, novel design concepts are possible to further improve functional ionic conducting materials.

\section{Author contributions}

The concept of the project was developed by T.B., M.T.A. and W.G.Z. Sample preparation, Xray diffraction, Rietveld refinements, sound velocity measurements and impedance analysis were performed by T.B. Data evaluation was done by T.B. and M.T.A. Two-channel modelling 
was performed by R.H. Nuclear magnetic resonance spectroscopy was carried out by B.W., whereas K.I. and S.L. carried out the high-temperature transport measurements. T.B. and M.P. measured the low-temperature transport properties. J.G. carried out the Harmonic force constant and Grüneisen parameter calculations. T.B., M.T.A. and W.G.Z. wrote the manuscript. All authors discussed the results and commented on the work.

\section{Acknowledgements}

The research as supported by the Deutsche Forschungsgemeinschaft (DFG) under grant number

ZE 1010/5-1. B.W. is member of the International Graduate School for Battery Chemistry, Characterization, Analysis, Recycling and Application (BACCARA), which is funded by the Ministry for Culture and Science of North Rhine Westphalia, Germany.We acknowledge access to various computational resources: The Tier-1 supercomputer of the Fédération WallonieBruxelles funded by the Walloon Region (grant agreement no. 1117545), and all the facilities provided by the Université catholique de Louvain (CISM/UCL) and by the Consortium des Equipements de Calcul Intensif en Fédération Wallonie-Bruxelles (CECI).

\section{References}

1. Janek, J. \& Zeier, W. G. A solid future for battery development. Nat. Energy 1, 1-4 (2016).

2. Liu, H. et al. Copper ion liquid-like thermoelectrics. Nat. Mater. 11, 422-425 (2012).

3. Lin, S. et al. High Thermoelectric Performance of $\mathrm{Ag}_{9} \mathrm{GaSe}_{6}$ Enabled by Low Cutoff Frequency of Acoustic Phonons. Joule 1, 816-830 (2017).

4. Li, B. et al. Liquid-like thermal conduction in intercalated layered crystalline solids. Nat. Mater. 17, 226-230 (2018).

5. Niedziela, J. L. et al. Selective breakdown of phonon quasiparticles across superionic 
transition in $\mathrm{CuCrSe}_{2}$. Nat. Phys. 15, 73-78 (2019).

6. Ding, J. et al. Anharmonic lattice dynamics and superionic transition in $\mathrm{AgCrSe}_{2}$. Proc. Natl. Acad. Sci. U. S. A. 117, 3930-3937 (2020).

7. Ziman, J. M. Electrons and Phonons. The Theory of Transport Phenomena in Solids. (Oxford University Press, 1963).

8. Hardy R.J. Energy-flux operator for a lattice. Phys. Rev. 132, (1963).

9. Seyf, H. R., Lv, W., Rohskopf, A. \& Henry, A. The importance of phonons with negative phase quotient in disordered solids. Sci. Rep. 8, 1-9 (2018).

10. Allen, P. B., Feldman, J. L., Fabian, J. \& Wooten, F. Diffusons, locons and propagons: Character of atomie yibrations in amorphous Si. Philos. Mag. B Phys. Condens. Matter; Stat. Mech. Electron. Opt. Magn. Prop. 79, 1715-1731 (1999).

11. Allen, P. B. \& Feldman, J. L. Thermal conductivity of disordered harmonic solids. Phys. Rev. B 48, 12581-12588 (1993).

12. Agne, M. T., Hanus, R. \& Snyder, G. J. Minimum thermal conductivity in the context of: Diffuson -mediated thermal transport. Energy Environ. Sci. 11, 609-616 (2018).

13. Hanus, R. et al. Transition from Crystal-like to Amorphous-like Heat Conduction in Structurally-Complex Crystals. 0-11 (2020) doi:10.26434/chemrxiv.12252056.

14. Simoncelli, M., Marzari, N. \& Mauri, F. Unified theory of thermal transport in crystals and glasses. Nat. Phys. 15, 809-813 (2019).

15. Ohno, S. et al. Materials design of ionic conductors for solid state batteries. Prog. Energy 2, 022001 (2020).

16. Boyce, J. B. \& Huberman, B. A. Superionic conductors: Transitions, structures, 
dynamics. Phys. Rep. 51, 189-265 (1979).

17. Culver, S. P., Krauskopf, T., Koerver, R. \& Zeier, W. G. Designing ionic conductors: the interplay between structural phenomena and interfaces in thiophosphatebased solidstate batteries. Chem. Mater. 30, 4179-4192 (2018).

18. Vargas-Barbosa, N. M. \& Roling, B. Dynamic Ion Correlations in Solid and Liquid Electrolytes: How Do They Affect Charge and Mass Transport? ChemElectroChem 7, $367-385$ (2020).

19. Bruesch, P., Pietronero, L., Strassler, S. \& Zeller, H. R. Brownian motion in a polarizable lattice: Application to superionic conductors. Phys. Rev. B 15, 4631-4637 (1977).

20. Qiu, P. et al. Suppression of atom motion and metal deposition in mixed ionic electronic conductors. Nat. Commun. 9, 4-11 (2018).

21. Shannon, R. D. \& Prewitt, C. T. Revised values of effective ionic radii. Acta Crystallogr. Sect. B Struct. Crystallogr. Cryst. Chem. 26, 1046-1048 (2002).

22. Li, W. et al. Low Sound Velocity Contributing to the High Thermoelectric Performance of $\mathrm{Ag}_{8} \mathrm{SnSe}_{6} . A d v . S c i .3$, (2016).

23. Jin, Z. et al. Abnormal thermal conduction in argyrodite-type $\mathrm{Ag}_{9} \mathrm{FeS}_{6-\mathrm{x}} \mathrm{Te}_{\mathrm{x}}$ materials. Mater. Today Phys. 19, 100410 (2021).

24. Heep, B. K. et al. High Electron Mobility and Disorder Induced by Silver Ion Migration Lead to Good Thermoelectric Performance in the Argyrodite $\mathrm{Ag}_{8} \mathrm{SiSe}_{6}$. Chem. Mater. 29, 4833-4839 (2017).

25. Shen, X. et al. High-Temperature Structural and Thermoelectric Study of Argyrodite $\mathrm{Ag}_{8} \mathrm{GeSe}_{6}$. ACS Appl. Mater. Interfaces 11, 2168-2176 (2019). 
26. Ohno, S. et al. How Certain Are the Reported Ionic Conductivities of ThiophosphateBased Solid Electrolytes? An Interlaboratory Study. ACS Energy Lett. 5, 910-915 (2020).

27. Kraft, M. A. et al. Influence of Lattice Polarizability on the Ionic Conductivity in the Lithium Superionic Argyrodites $\mathrm{Li}_{6} \mathrm{PS}_{5} \mathrm{X}(\mathrm{X}=\mathrm{Cl}, \mathrm{Br}, \mathrm{I})$. J. Am. Chem. Soc. 139, 10909-10918 (2017).

28. Krauskopf, T. et al. Comparing the Descriptors for Investigating the Influence of Lattice Dynamics on Ionic Transport Using the Superionic Conductor $\mathrm{Na}_{3} \mathrm{PS}_{4-\mathrm{x}} \mathrm{Se}_{\mathrm{x}} . J$. Am. Chem. Soc. 140, 14464-14473 (2018).

29. Sales, B. C., Mandrus, D. G. \& Chakoumakos, B. C. Chapter 1 Use of atomic displacement parameters in thermoelectric materials research. Semiconductors and Semimetals vol. 70 (2001).

30. Hanus, R. et al. A Chemical Understanding of the Band Convergence in Thermoelectric $\mathrm{CoSb}_{3}$ Skutterudites: Influence of Electron Population, Local Thermal Expansion, and Bonding Interactions. Chem. Mater. 29, 1156-1164 (2017).

31. Christensen, M. et al. Avoided crossing of rattler modes in thermoelectric materials. Nat. Mater. 7, 811-815 (2008).

32. Hanus, R. et al. Lattice Softening Significantly Reduces Thermal Conductivity and Leads to High Thermoelectric Efficiency. Adv. Mater. 1900108 (2019) doi:10.1002/adma.201900108.

33. Toberer, E. S., Zevalkink, A. \& Snyder, G. J. Phonon engineering through crystal chemistry. J. Mater. Chem. 21, 15843-15852 (2011).

34. Tan, G. et al. High thermoelectric performance $\mathrm{SnTe}-\mathrm{In}_{2} \mathrm{Te}_{3}$ solid solutions enabled by 
resonant levels and strong vacancy phonon scattering. Chem. Mater. 27, 7801-7811 (2015).

35. Voneshen, D. J. et al. Suppression of thermal conductivity by rattling modes in thermoelectric sodium cobaltate. Nat. Mater. 12, 1028-1032 (2013).

36. Yelon, A., Movaghar, B. \& Crandall, R. S. Multi-excitation entropy: Its role in thermodynamics and kinetics. Reports Prog. Phys. 69, 1145-1194 (2006).

37. Muy, S. et al. Lithium Conductivity and Meyer-Neldel Rule in $\mathrm{Li}_{3} \mathrm{PO}_{4}-\mathrm{Li}_{3} \mathrm{VO}_{4}-$ $\mathrm{Li}_{4} \mathrm{GeO}_{4}$ Lithium Superionic Conductors. Chem. Mater. 30, 5573-5582 (2018).

38. Yelon, A. \& Movaghar, B. Microscopic explanation of the compensation (MeyerNeldel) rule. Phys. Rev. Lett. 65, 618-620 (1990).

39. Muy, S., Schlem, R., Shao-Horn, Y. \& Zeier, W. G. Phonon-Ion Interactions: Designing Ion Mobility Based on Lattice Dynamics. Adv. Energy Mater. 2002787 (2020).

40. Wang, Y. et al. Design principles for solid-state lithium superionic conductors. Nat. Mater. 14, 1026-1031 (2015).

41. Gordiz, K., Muy, S., Zeier, W. G., Shao-Horn, Y. \& Henry, A. Enhancement of ion diffusion by targeted phonon excitation. Cell Reports Phys. Sci. 2, 100431 (2021).

42. Lin, S., Li, W. \& Pei, Y. Thermally insulative thermoelectric argyrodites. Mater. Today, 40-45 (2021).

43. Cahill, D. G., Watson, S. K. \& Pohl, R. O. Lower limit to the thermal conductivity of disordered crystals. Phys. Rev. B 46, 6131-6140 (1992).

44. Jin, M. et al. Fabrication and Thermoelectric Properties of Single-Crystal Argyrodite $\mathrm{Ag}_{8} \mathrm{SnSe}_{6}$. Chem. Mater. 31, 2603-2610 (2019). 
45. Hanus, R. et al. Thermal transport in defective and disordered materials.

46. Brenner, T. M. et al. Anharmonic host-lattice dynamics enable fast ion conduction in superionic AgI. Phys. Rev. Mater. 4, 115402 (2020).

47. Seyf, H. R. \& Henry, A. A method for distinguishing between propagons, diffusions, and locons. J. Appl. Phys. 120, (2016).

48. Takahashi, T., Yamamoto, O., Matsuyama, F. \& Noda, Y. Ionic conductivity and coulometric titration of copper selenide. J. Solid State Chem. 16, 35-39 (1976).

49. Zhang, Z. et al. $\mathrm{Cu}_{2} \mathrm{Se}$-Based liquid-like thermoelectric materials: Looking back and stepping forward. Energy Environ. Sci. 13, 3307-3329 (2020).

50. Krishnamoorthy, A. et al. Optical Control of Non-Equilibrium Phonon Dynamics. Nano Lett. 19, 4981-4989 (2019). 